\title{
On the Relationship of the Discrete Model of the Nuclei of Linear and Planar Defects and the Continuum Models of Defects in Crystalline Materials
}

\author{
V. L. Busov \\ Donbass State Engineering Academy, Kramatorsk, Ukraine \\ Email:vlbusov49@gmail.com
}

How to cite this paper: Busov, V.L. (2020) On the Relationship of the Discrete Model of the Nuclei of Linear and Planar Defects and the Continuum Models of Defects in Crystalline Materials. Applied Mathematics, 11, 862-875.

https://doi.org/10.4236/am.2020.119056

Received: June 17, 2020

Accepted: September 1, 2020

Published: September 4, 2020

Copyright $\odot 2020$ by author(s) and Scientific Research Publishing Inc. This work is licensed under the Creative Commons Attribution International License (CC BY 4.0).

http://creativecommons.org/licenses/by/4.0/

\begin{abstract}
A physical and mathematical model of the transition from a discrete model of linear and flat defects nuclei to continuum models of defects such as dislocations and disclinations and their combinations is presented, where the tensors of energy-momentum and angular momentum of an alternating field are considered, for which the type and structure of the Maxwell stress tensor $\sigma_{\alpha \beta}^{i f}$ are given and the corresponding angular momentum tensor, using the dynamic equation for the evolution of internal stresses and the correlation between the stresses $\sigma_{\alpha \beta}^{i f}$ in the defect core and the elastic stresses $\sigma_{i k}^{e l}$ in its environment, obtains elastic displacement and deformation fields identical to these fields from Burgers and Frank vectors of continuous models. The spectral density of the autocorrelation functions of the velocity of photoelectrons $\Psi_{\perp}^{e}(\beta)$ and cations $\Psi_{\perp}^{\text {cat }}(\beta)\left(\beta=\hbar \omega_{\mu} / k T ; \mu \equiv e, c a t\right)$, which transforms into linear spectra as $T \rightarrow 0$, is considered reflecting the existence of threshold values of oscillation and rotations currents of photoelectrons and cations at all stages of plastic deformation and fracture. The features of the process of sliding linear defects in metals are disclosed.
\end{abstract}

\section{Keywords}

Maxwell Stress Tensor of an Alternating (Intermittent) Field, Equation of Evolution of Internal Stresses, Autocorrelation Function of the Speed of Photoelectrons and Cations 


\section{Introduction}

Currently, there are several definitions of linear defects in crystalline materials:

1) The phenomenological definition of an edge dislocation [[1] [2] p. 235] includes the insertion of an extra half-plane, forming regions of condensation and rarefaction of atoms above and below the slip plane with a normal $\boldsymbol{n}$. Its edge inside the crystal corresponds to a dislocation line with the unit vector $\tau$, and the Burgers vector is equal to the sum of the increments $\sum_{i}^{N} \Delta \boldsymbol{u}_{i}$ of the atom displacement vector $\boldsymbol{u}_{i}$ in a closed loop around the dislocation line for $\boldsymbol{b}_{e d} \perp \boldsymbol{\tau}$ and $\boldsymbol{b}_{e d} \perp \boldsymbol{n}$. The bending and turn around the $\tau$ оси axis of crystallographic planes whose normals are parallel to $\tau$ form a screw dislocation, where the Burgers vector $\boldsymbol{b}_{s c r}=\sum \Delta \boldsymbol{u}_{i}$, but $\boldsymbol{b}_{s c r} \| \boldsymbol{\tau}$ is similar.

2) In the framework of the theory of an elastic continuum modeling a medium surrounding a dislocation core [2] [3], the well-known procedure is applied: the crystal is cut along the slip plane, the dislocation core is removed, the cut surfaces of the resulting dislocation tube are shifted along the slip plane in the corresponding direction, forming a boundary or screw dislocations with the Burgers vector $\boldsymbol{b}_{\gamma}=\oint \mathrm{d} u_{i} ; \gamma \equiv e d g, s c r$. We note that here the dislocation line is the line of singular points, where the discontinuity vector shifts $\left[\boldsymbol{u}_{i}\right]=\boldsymbol{b}_{\gamma}$.

3) To describe the boundaries of tilt and torsion using a model representation-disclination as an element of rotational plasticity [[3] p. 254]. Here the determining parameter is the rotation vector $\omega$, whose module is equal to the misorientation angle of blocks, subgrains, fragments, while the disclination line serves as the axis of rigid rotation of the subgrains and on $[\boldsymbol{u}]=\boldsymbol{\Omega} \times \boldsymbol{r}, \boldsymbol{\Omega}$ is the Frank vector- $\theta$. In the phenomenological definition of disclination, the Frank vector $\Omega$ is introduced, equal to $-\sum_{i}^{N} \Delta \omega_{i}$ when traversing a closed loop around the disclination line. The inclination disclination is a model analogue of the dislocation wall for $\Omega \| \tau$, the torsion disclination is the torsion boundary with the normal $\boldsymbol{n}$ for $\Omega \| \boldsymbol{n}$.

Numerous experimental results using the methods of ion design, X-ray topography, electron microscopy, moire [[4] p. 36; [5] p. 323] basically confirm the phenomenological and continuum definitions of linear defects. At the same time, due to the insufficient resolution of the above methods (not less than $0.6 \div 0.8$ $\mathrm{nm}$ ), it has not yet been possible to directly observe the structure of the defect nucleus except refractory metals such as molybdenum and crystals of copper and platinum phtalocyanide.

It is known that the structure of the usual boundaries of tilt and torsion is determined by the method of relaxation tuning of atoms, which form an interlayer with a thickness of $2-3$ atomic layers with a minimum excess energy of the boundary [6]. In the liquid boundary model [7], the crystal structure is completely broken and can be represented as a combination of vacancies and disordered atoms. A number of other works [8] [9], based on a symmetric tilt boundary in the form of a lattice dislocation wall and the Reed-Shockley formula, consider the amorphization process, where the nuclei of these lattice dislocations 
merge at critical misorientation angles into an amorphous core of the boundary. Within the framework of the general theory of special boundaries in a crystal [6], N. Mott [10] proposed a compromise version of such adjustment to special boundaries in the island model, where a periodic sequence of regions of poor and good conjugation of atoms of bicrystal lattices is presented.

It is known [11] that near the interface "liquid-gas" in the transition layer "liquid metal-gas" stable density oscillations $\rho(z)$ arise [[11], p. 156] with a spatial period $a_{0}$, the longitudinal autocorrelation function $\Psi_{\perp}^{a t}(t)=\dot{z}(t) \cdot \dot{z}(0) /\left\langle\dot{z}^{2}(0)\right\rangle$ the velocities of atoms for moving along the normal $z$ to the surface of the liquid are oscillatory quasicrystalline in time scale with a period equal to the lifetime of surface phonons of the order of $4.5 \times 10^{-12} \mathrm{~s}$, and the spectral density $\Psi_{\perp}^{a t}(\beta)$ on the energy dimensionless scale $\beta=\hbar \omega / k T$ has two pronounced peaks and three - four less pronounced peak with an exponential envelope for the entire series of peaks above the background, the area of which is also limited by the exponent [[11], p. 254].

Starting from the works of G. Weingarten, A. Somigliana, and V. Volterra, continuum dislocation models are based on physical and mathematical abstraction in the form of a line endowed with mass, velocity, energy, linear tension and the Burgers vector, where the degree of physicality of the model is not large enough compared to its mathematics; as in the theory of linearly deformed media, only Hooke's physical law holds. A natural question arises: What is the structure of the nuclei of linear and planar defects in the language of charges and their currents, because nothing except them exists in crystalline materials? How does this structure reflect the dynamic processes of generation and slip of such defects?

The aim of this work is to build a transitional model from a discrete model of linear and flat defects nuclei to continuum dislocation-disclination models of crystalline materials.

\section{The Theoretical Model}

First, note that the continual dislocation-disclination models have two important features of the mathematical apparatus:

1) when describing translational plasticity, the Burgers vector is introduced on the defect line, and when describing rotational plasticity, the Frank vector;

2 ) in these models, in most cases, they consider a static problem using equilibrium equations, where a solution is sought using the Green's function $G_{j k}^{s t}$, the regular component of which is long-range: $\hat{G}_{r e g} \sim r^{-1}$ ( $r$ is the distance from the defect line to the point $r$ ) [[12], p. 379].

In the framework of the discrete model of the nuclei of linear defects [13], the general structure of the solutions of the wave equation of the alternating field as an analog of the electromagnetic field using the Green's functions [[14], p. 77] in the form of a vector potential

$$
A_{\mu}^{i f}(x)=\int G_{\mu v}^{i f}\left(x-x^{\prime}\right) \sum_{v} I_{\nu}^{k} \mathrm{~d}^{4} x^{\prime}(v=e, c a t ; x \equiv \boldsymbol{r}, t),
$$


where the components of the tensor of the Green's function in the generalized space of rectangular momenta have the form

$$
G_{\mu \nu}^{i f}(\boldsymbol{r}, t)=G_{\mu \nu+}^{i f}(\boldsymbol{r}) \cdot \theta(t)+G_{\mu \nu-}^{i f}(\boldsymbol{r}) \cdot \theta(-t),
$$

and the functions $\theta(t)$ and $\theta(-t)$ are combinations of Heaviside stepwise unit functions of the type $[U(t)-U(t-\tau)]$ [13], leading to rectangular pulses of various durations $\tau$ or approximated in shape to rectangular ones; the functions $G_{\mu \nu+}^{i f}(\boldsymbol{r})$ and $G_{\mu \nu-}^{i f}(\boldsymbol{r})$ contain regular and singular components [[14], p. 71]

$$
G_{\mu \nu \pm}^{i f}(\boldsymbol{r})=\frac{1}{(2 \pi)^{2} \cdot r^{2}} \mp \frac{1}{4 \pi} \delta\left(r^{2}\right)
$$

and describes, respectively, a wave running to the right along the defect line or a wave reflected from its left edge of the defect fixing nodes and a wave running to the left or reflected from the right edge of the defect. According to [13], one of the possible schemes for generating linear defect nuclei includes the photoelectric effect of the electrons of the inner shells of cations as a result of resonant scattering of primary photons of an intermittent (alternating) field and the formation of long-wave secondary photons that are elastically scattered in the region of the defect. The energy of primary photons $\varepsilon_{\gamma}$ is redistributed between the transition energy $\varepsilon_{i j}$ of photoelectrons between discrete states $i \rightarrow j$ in the matrix cations, the kinetic energy of photoelectrons $\varepsilon_{\text {phe }}$ knocked out of cations, and the energy of secondary photons $\varepsilon_{\text {sph }}$, which mainly determine the elastic deformation fields and stresses of a good crystal. The kinetic energy of photoelectrons $\varepsilon_{\text {phe }}$ is completely determined by the pulse density of the alternating field $E_{j}^{i f}$ or the three-dimensional tensor $\sigma_{\alpha \beta}^{i f}$ of the Maxwell stresses as part of the four-dimensional energy-momentum tensor $T_{\mu v}^{i f}$ of the field $E_{j}^{i f}$.

The system of pairs of photoelectrons and cations in the defect nucleus has two degrees of freedom: oscillations and rotations per charged particle, therefore, in addition to $T_{\mu \nu}^{\text {if }}$, we must use the angular momentum tensor $M_{\mu \nu}^{\text {if }}$ of the field $E_{j}^{\text {if }} \quad\left[[14]\right.$, p. 26]. Note that the values of $\varepsilon_{i j}$ correspond to atomic spectra and remain unchanged in time and space. Hence the statement about the correlation between the components of the elastic internal stress tensors $\sigma_{m n}^{e l}$ in the medium surrounding the defect core (good crystal) and $\sigma_{\alpha \beta}^{i f}$ in the defect core (bad crystal) has a right to exist.

Let us briefly disclose a scheme of the mechanism of influence of secondary long-wavelength photons of an alternating field on the environment surrounding the core of a defect. According to atomic spectra, these photons cannot produce resonant scattering by cations at lattice sites, but they carry out elastic scattering by them. In addition, they are bosons, obey wave-particle duality, their density decreases from the defect line according to the hyperbolic law. Here, the elastic scattering of photons by cations does not change the internal state of cations, but when they are backscattered by large angles from $\pi / 2$ to $\pi$, within the 
framework of the momentum conservation law, cations acquire recoil momentum $\boldsymbol{p}_{\text {cat }}^{\text {rec }}$, leading to elastic displacements from lattice sites. Note that, in this case, the directions of the polarization vectors of the primary and secondary photons are close or coincide, which leads to the coincidence of the types of tensor matrices $\sigma_{j k}^{i f}$ and $\sigma_{l m}^{e l}$. Apparently, this is precisely the physical essence of Hooke's law in quantum electrodynamics. In addition, during elastic scattering, the cation nuclei acquire the radial components $\boldsymbol{p}_{\text {cat }}^{\text {rec }}$ from the defect line, which corresponds to the radial elastic deformation of all-round tension, but they can also acquire the tangential components $\boldsymbol{p}_{\text {cat }}^{\text {rec }}$ normal to the defect line and to the field polarization vector $E_{j}^{i f}$, which in turn corresponds to tangential elastic cation deformation or shear deformation. From this, the correlation between $\sigma_{j k}^{i f}$ and $\sigma_{l m}^{e l}$ becomes clear.

Here we can apply the dynamic equation of evolution of internal stresses $\sigma_{m n}^{e l}$ [15] [16], the members of the right side of which describe the correlation between the components of the tensor $\sigma_{m n}^{e l}$, between the components $\sigma_{m n}^{e l}$ and $\sigma_{\alpha \beta}^{i f}$, the tensor of external stresses $\sigma_{i k}^{\text {out }}$

$$
\frac{\mathrm{d} \hat{\sigma}^{e l}}{\mathrm{~d} t}=-\lambda^{e l} \cdot \hat{\sigma}^{e l}+\alpha \cdot\left(\hat{\sigma}^{e l}\right)^{2}+\gamma \cdot \hat{\sigma}^{e l} \cdot \hat{\sigma}^{i f}+\eta \cdot \hat{\sigma}^{e l} \cdot \hat{\sigma}^{\text {out }},
$$

where $\lambda^{e l}=\tau_{\text {rcat }}^{-1}, \tau_{\text {rcat }}$ is the fast relaxation time of cations in a good crystal. When considering such a correlation, the structural-phase transition in the region between a good and a bad crystal becomes important. Here in the potential relief $u(\boldsymbol{r}, t)$ its microscopic component $u^{\prime}(\boldsymbol{r}, t)$, fluctuating over the times $t \ll \tau_{D} \quad\left(\tau_{D}\right.$ is the Debye time), becomes comparable with its macroscopic component $U(\boldsymbol{r}, t))$ for $t \gg \tau_{D}$. The intermittent field reduces the point symmetry of the perfect crystal to axial, thereby creating its dynamic anisotropy in the direction of the field, which essentially forms the corresponding distribution of atom-vacancy displacements. This allows us to draw an analogy between the longitudinal autocorrelation function of the velocity of atoms $\Psi_{\perp}^{a t}(t)$ on the liquid-gas surface and the same function of atoms, photoelectrons $\Psi_{\perp}^{e}(t)$ and cations $\Psi_{\perp}^{\text {cat }}(t)$ in the radial direction from the defect line in the transition region, taking into account the Coulomb attraction.

It should be noted here that at absolute temperature $T \rightarrow 0$, all peaks of the spectral density $\Psi_{\perp}^{a t}(\beta) \quad(\beta=\hbar \omega / k T), \Psi_{\perp}^{e}(\beta), \Psi_{\perp}^{\text {cat }}(\beta)$ transform into $\delta$-functions Dirac, while the intervals between neighboring peaks increase by two orders of magnitude with a decrease from room to helium temperatures, the exponential envelope of the peaks tends to the asymptote in the form of a straight line parallel energy scale $\beta$, and the background component goes into a narrow band adjacent to the scale $\beta$. In other words, $\Psi_{\perp}^{a t}(\beta), \Psi_{\perp}^{e}(\beta)$, $\Psi_{\perp}^{\text {cat }}(\beta)$ turn into line spectra, each line of which, apparently, characterizes the time-separated processes of translational, rotational plasticity, fragment formation, microcracks and their merging at all stages of plasticity and fracture.

An analysis of expressions (1)-(4) shows that the dependence of the components $\sigma_{m n}^{e l}(r)$ is long-range and is determined by the shape and size of the va- 
cancy volume, the nature of the distribution of the oscillation and rotations currents of photoelectrons and cations in the nuclei of linear and plane defects. In the generalized space of defect nuclei, the amplitude values of $\sigma_{m n}^{e l}$ are determined by the amplitude and duration of the rectangular pulses of the alternating field [13].

\subsection{Edge Dislocation}

We direct the $z$ axis of the rectangular coordinate system along the line of the edge dislocation, the slip plane is compatible with the $x 0 z$ plane with the normal $\boldsymbol{n}$, and the $y$ axis along $\boldsymbol{n}$. In the theory of individual dislocations, the elastic stress tensor $\sigma_{m n}^{e l}$ in the medium surrounding the core of the edge dislocation has a $2 \times 2$ matrix [[17], p.57]

$$
\hat{\sigma}_{e d g}^{e l}=\left(\begin{array}{cc}
\sigma_{x x} & 0 \\
\tau_{y x} & \sigma_{y y}
\end{array}\right),
$$

which corresponds to plane deformation at $u_{z}=0$. Here, the tangent component $\tau_{y x}$ creates a shearing in the $y 0 z$ plane in the positive or negative direction of the $y$ axis. In the core of the dislocation, an alternating field forms linear structures along the $y$ axis, moreover, vacancy chains appear in parallel electronic chainseither below the slip plane parallel to the $z$ axis, where cationic chains are located from last at a distance $b_{0}$, and electronic chains are located at a distance $\leq 2 b_{0}$ from dislocation line, or above the slip plane, etc. The Maxwell stress tensor $\hat{\sigma}_{\text {edg }}^{i f}$ preserves the type of matrix (5)

$$
\hat{\sigma}_{e d g}^{i f}=\left(\begin{array}{cc}
\sigma_{x x}^{i f} & \tau_{x y}^{i f}\left(E_{x}^{o w n}\right) \\
\tau_{y x}^{i f}\left(E_{y}^{o w n}\right) & \sigma_{y y}^{i f}
\end{array}\right),
$$

where the tangent components $\tau_{x y}^{i f}$ and $\tau_{y x}^{i f}$ contain only the real parts, depending on the components of the field $E_{j}^{\text {own }}$ in the $x 0 y$ plane, the imaginary parts of these components [13] are equal to zero. Here, the $\tau_{x y}^{i f}$ component determines the slip process under the influence of $E_{x}^{\text {own }}$ and $E_{x}^{\text {out }}$, and the component $\tau_{y x}^{i f}$ determines the generation of the dislocation core and creep under the influence of $E_{y}^{\text {own }}$ and $E_{y}^{\text {out }}$. Note that the arrangement of the indices of the tensors $\sigma_{\alpha \beta}^{i f}$ and $\sigma_{i k}^{e l}$ in this work is carried out according to field theory [[18], p. 109], where the first index determines the direction of action of the force or density of the momentum flux, and the second, the normals to the site of application of force or to flow surface. For the edge dislocation, the components of the angular momentum tensor of the alternating field are $\hat{M}_{\text {edg }}^{\text {if }}=0$.

\subsection{Screw Dislocation}

Let us consider one of the possible schemes for generating linear structures in the core of a screw dislocation. Here, the formation of linear structures occurs in two mutually perpendicular glide planes, while the vacancy chain is formed on the dislocation line by means of four such structures, as shown in Figure 1 . 

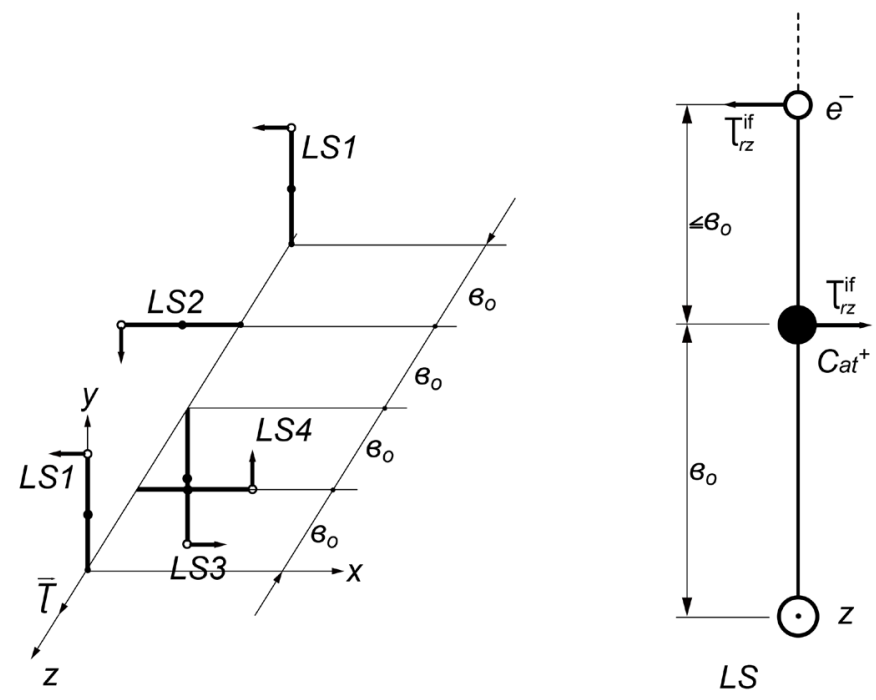

Figure 1. Scheme of the formation of linear structures LS1, LS2, LS3, LS4 and application of the Maxwell intermittent field stresses $T_{j k}^{i f}$ on charged particles in the core of a screw dislocation in metals with face-centeredlattice.

Transverse photons of the alternating field form the LS1, 2, 3, 4 structures. Quantum electrodynamics allows the existence of longitudinal photons [[14], p. 62], corresponding to the $z$-component of the field $z$-along the dislocation line. We introduce the threshold values of the rotational currents of the photoelectrons $J_{\text {eturn }}^{\text {thr }}$ and cations $J_{\text {catturn }}^{\text {thr }}$. If the photoelectron current is $J_{e}<J_{\text {eturn }}^{\text {thr }}$, then photoelectrons perform torsional oscillations near the cations from which they were knocked out, and cations also make similar oscillations from the influence of currents with an amplitude three orders of magnitude smaller than $J_{e}$. Hence, the threshold values $J_{\text {eturn }}^{\text {thr }}$ and $J_{\text {catturn }}^{\text {thr }}$ are connected by a simple relation

$$
J_{\text {catturn }}^{\text {thr }} \approx \frac{2 m_{e}}{M_{\text {cat }}} J_{\text {eturn }}^{\text {thr }} .
$$

For $J_{e}=J_{\text {eturn }}^{\text {thr }}$, the time dependence of $J_{e}(t)$ in the generalized space of rectangular pulses is shown in Figure 2. Here, the descending section of an individual pulse on the curve $J_{e}(t)$ arises under the influence of the driving force $F_{j}^{f}\left(E_{j}^{o w n}, H_{j}^{\text {own }}\right)$ in the form of the Dirac $\delta$-function at $t=0$ and the restoring force $F_{j}^{r e s t}$ from the Coulomb attraction of the cation. The current $J_{e}$ includes the transition current $J_{e}^{t r}$, the oscillation and rotation current $J_{e}^{v}$, and the displacement current along the dislocation line under the influence of $E_{z}^{i f}$. The trajectory of photoelectrons at $J_{e}>J_{\text {eturn }}^{\text {thr }}$ and $E_{z}^{o w n}>E_{z}^{\text {thr }}$ ( $E_{z}^{\text {thr }}$ is the threshold field value for the displacement of the photoelectron from the cation in LS1 to the cation in LS2, etc.) takes the form of a spiral around $z$ axis. The cations in the core of the dislocation perform only torsional oscillations. Such a structure of pairs of photoelectrons and cations in the nucleus of a screw dislocation and the trajectory of motion of charged particles allows us to write the components of the Maxwell stress tensor $\sigma_{\alpha \beta}^{i f}$ in the form of a $3 \times 3$ matrix 


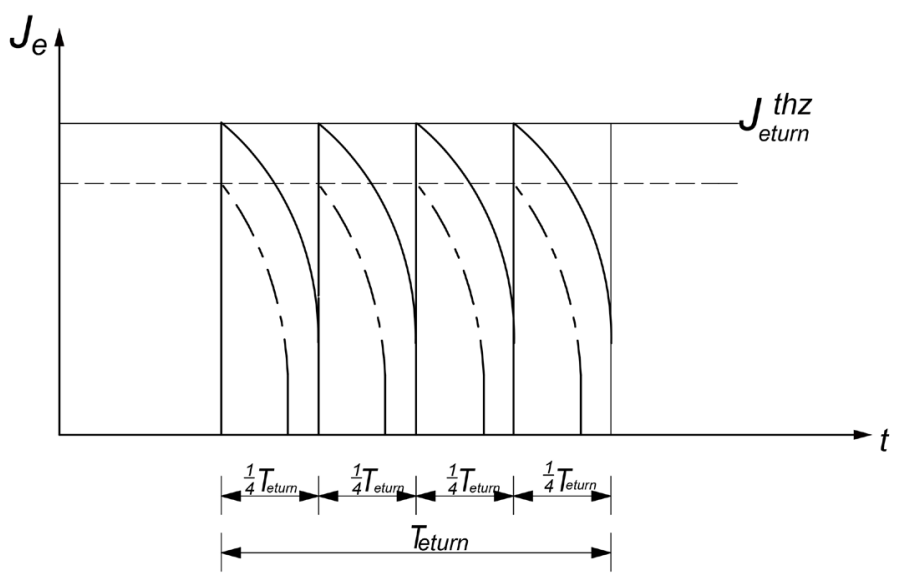

Figure 2. Time dependence of the photoelectron current $J_{e}$ in a screw dislocation core, $J_{\text {eturn }}^{\text {thr }}$ is the threshold value of the photoelectron rotation current.

$$
\sigma_{\alpha \beta}^{i f}=\left(\begin{array}{ccc}
\sigma_{x x}^{i f}\left(E_{x}^{o w n}\right) & 0 & \tau_{x z}^{i f}\left(i H_{z}^{o w n}\right) \\
0 & \sigma_{y y}^{i f}\left(E_{y}^{o w n}\right) & \tau_{y z}^{i f}\left(i H_{z}^{o w n}\right) \\
0 & 0 & \sigma_{z z}^{i f}\left(E_{z}^{o w n}\right)
\end{array}\right),
$$

which reflects the axial symmetry of the field of elastic strains and stresses $\sigma_{\alpha \beta}^{e l}$ in a good screw dislocation crystal. In (8) $\frac{\sigma_{x x}^{i f}+\sigma_{y y}^{i f}+\sigma_{z z}^{i f}}{3}=-p$, and $\sigma_{x x}^{i f}=\sigma_{y y}^{i f}=\sigma_{z z}^{i f}=-p$, which corresponds to all-round extension on the screw dislocation line, and the components vectors $H_{j}^{\text {own }}=\left(0 ; 0 ; H_{z}^{\text {own }}\right)$. Here the components of the angular momentum tensor $M_{i k}^{i f}$ represent the antisymmetric tensor of the second rank

$$
M_{i k}^{i f}=\left(\begin{array}{ccc}
0 & 0 & \pm \tau_{x z}^{i f} \cdot y \\
0 & 0 & \pm \tau_{y z}^{i f} \cdot x \\
0 & 0 & 0
\end{array}\right),
$$

where for structures LS1 and LS3 the combination of signs is $\pm \tau_{x z}^{i f} \cdot y$ and $\mp \tau_{x z}^{i f} \cdot(-y)$ respectively, and for structures LS2 and LS4: $\pm \tau_{y z}^{i f} \cdot x$ and $\mp \tau_{y z}^{i f} \cdot(-x)$ (Figure 1). The combinations of signs before the products of stress and coordinate values reflect, respectively, the left-sided and right-sided rotation systems with respect to the unit vector $\tau$. We assume that the probabilities of the appearance of right-handed and left-handed rotation systems are the same and equal to $1 / 2$.

\subsection{Disclosure Models}

Experience shows [19] [20] that with large plastic deformations, translational plasticity is replaced by rotational plasticity, in the description of which various types of disclination are introduced. One of the main features of the structure of the tilt and torsion boundaries is the combination of vacancy chains of several 
linear structures into a single bundle, the transformation of vacancy clusters into vacancy volumes in the form of disks, drops, dumbbells, and rectangular stripes. Here, in the generalized space of rectangular pulses, the simultaneous generation of rotations of cations and photoelectrons with respect to the cation chains from which they were knocked out begins to play a role. The existence of closed trajectories of rotations of cations and photoelectrons around vacancy volumes for $J_{\text {cat }}>J_{\text {catturn }}^{\text {thr }}$ and $J_{e}>J_{\text {eturn }}^{\text {thr }}$, taking into account the Coulomb attraction, can ensure their stability in space and time for any kind of defects, both linear and plane. Here are the criteria that allow us to build discrete models of the nuclei of the tilt and torsion boundaries with rotational plasticity:

1) Energy criterion. The energy of the currents of photoelectrons and cations from which the photoelectrons were knocked out should correspond to the minimum elastic energy of the crystal in the medium surrounding the defect core;

2) Geometric criterion. The motion paths of the above photoelectrons and cations should be within the layer $3-4 a_{0}$;

3) The fulfillment of the electroneutrality condition both for the subsystems of photoelectrons and cations, and for conduction electrons and matrix cations;

4) The absence of long-range fields of internal stresses from the boundaries of tilt and torsion.

Consider the form of vacancy volumes $V_{\text {vac }}$ for various types of disclinations. Here we will use the disclinations formation schemes in the elastic continuum model [[20], p. 30]. For a wedge disclination, $V_{\text {vac }}$ is located asymmetrically to the defect line along the $z$ axis, has an axial section in the shape of a rolling profile of T-beam within the wedge angle, where the wall of the T-beam is located in the radial direction, and its shelf is parallel to the $z$ axis. To disclose torsion with the rotation vector $\boldsymbol{\Omega} \perp \boldsymbol{\tau}$, the volume $V_{\text {vac }}$ takes the form of an I-beam, the wall of which is parallel to the $z$ axis, and the shelves are axisymmetric with it. The volume $V_{v a c}$ for general disclination approaches in shape to a dumbbell axisymmetric with the defect line, and its axial sections are elliptical.

\subsection{Translational Ductility in Metals}

A detailed quantitative description of the transition model is possible by numerically calculating the Vlasov system of equations, the wave equation of the alternating field, and the equation of evolution of internal stresses, which requires separate work. In this paper, we restrict ourselves to the consideration of translational plasticity of metallic crystals with face centered lattice and estimate the threshold values of the photoelectron oscillation current $J_{\text {eosc }}^{\text {thr }}$ of the onset of slip of a screw dislocation in copper. Here, the process of knocking out and moving the photoelectrons is essentially an internal photoelectric effect, where the kinetic energy received from the field $E_{j}^{i f}$ must not be lower than the sum of the work of the returning forces $F_{j}^{\text {rest }}$ of the cation from which it was knocked out, of three pairs of its nearest neighbors along the cation chain and 
the forces of electrostatic interaction from the side of the excess charge distribution $q(\rho)\left[\mathrm{cm}^{-3}\right]$ conduction electrons [21] in copper

$$
q(\rho)=\beta \cdot \frac{8 \pi}{3} \sqrt{\frac{k_{F}}{\pi}} \cdot A \cdot \frac{\cos \left(2 k_{F} \rho+\Phi\right)}{\rho^{5 / 2}},
$$

where the parameters $\beta=7.62 ; A=0.052 ; \Phi=0.1015$ are taken from [21]; $k_{F}$ is the Fermi wave number, for copper $k_{F}=1.36 \times 10^{8} \mathrm{~cm}^{-1}$ [[22], p. 260], as a result of the screening of the deformation induced by the long-range field $\varepsilon \sim \rho^{-1}$, for overcoming the first four most significant concentric layers $q(\rho)$ with a variable sign [21]

$$
\frac{m_{e} \cdot v_{0 x}^{2}}{2}-\frac{m_{e} \cdot v_{f x}^{2}}{2}=\sum_{i}^{3} A_{i}=\sum_{i}^{3} \sum_{j}^{3} F_{j} \cdot l_{j} \cdot \cos \gamma_{i} \cdot \cos \alpha,
$$

where the change in the kinetic energy of the photoelectrons obtained from the field $E_{j}^{i f}$ is equal to the work of the sum of the Coulomb interaction forces from the point charges of the nuclei of the donor cations and the three pairs of nearest neighbors along the cation chain and the distribution $q(\rho) ; l_{j}$ is the photoelectron displacement vector along the $x$ axis; $\gamma_{i}$ is angle between the directions of the vectors $F_{j}$ and $l_{j} ; \alpha$ is the angle between the direction of the Coulomb force and the $x$ axis, $\alpha=\pi-\gamma$. The distribution $q(\rho)$ is considered in a cylindrical coordinate system, and the photoelectron displacement is in the Cartesian coordinate system, whose $z$ axes coincide. For calculation, we use the standard approach for describing [23] the motion of charged particles in an electromagnetic field for various systems of interacting charges. We assume that the nonrelativistic approximation is applicable here, and the radiation of moving particles is absent. Note that in the calculation, the dielectric constant $\varepsilon_{m}(\boldsymbol{k}, \omega)$ acquires an important influence from the non-stationary effect of the alternating field with the pulse repetition rate $\omega_{\text {rep }}[24]$

$$
\varepsilon_{m}(\boldsymbol{k}, \omega)=1-\frac{\omega_{p}^{2}}{\omega_{\text {rep }}^{2}},
$$

where $\omega_{p}$ is the plasma frequency of the subsystem of conduction electrons [25], $\omega_{p}=\left(4 \pi e^{2} \cdot n_{0} / m_{e}\right)^{1 / 2} ; n_{0}$ is the density of conduction electrons $\left[\mathrm{cm}^{-3}\right]$, where for copper $n_{0}=8.45 \times 10^{22} \mathrm{~cm}^{-3}[22] ; m_{e}=9.11 \times 10^{-28} \mathrm{~g} ; e=4.8 \times 10^{-10}$ CGSE $_{\mathrm{q}}$ [26]. If $\omega_{\text {rep }}$ is $10^{14} \div 10^{15} \mathrm{~s}^{-1}$, and $\omega_{p}=1.64 \times 10^{16} \mathrm{ceK}^{-1}$, then according to (12) $\left|\varepsilon_{m}(\boldsymbol{k}, \omega)\right|=10^{2} \div 10^{4}$. At the first stage of the calculation, we use the fact that the electric field outside the infinitely long circular cylinder uniformly charged in volume or on the surface is identical to the field of the infinite uniformly charged line $E_{\text {line }} \sim \chi \cdot \rho^{-1}$, where $\chi$ is the linear charge density, and the field inside hollow cylinder is zero [[23] [27], p. 232]. In our case, the distribution $q(\rho)$ can be divided into a number of layers of an excess charge of variable sign according to the law cos. For an arbitrary cylindrical surface with a radius $\rho=x$ inside the $n$-layer, the charge $q(\rho)$ is placed in two tubes: the upper and lower. We apply the principle of superposition to charges in tubes. For $\rho>x$, all $(n-1)$-layers and the lower tube create a field outside the $n$-layer, 
and for $\rho \leq x$ the field from the upper tube is zero. Hence, the electric field acting on the photoelectron from the side $q(\rho)$ is represented as the sum

$$
E(x)=\sum_{n=1}^{n}\left[E_{n+1}(x)+\Delta E_{n l}(x)\right],
$$

where the external fields $E_{n}(x)$ and $\Delta E_{n l}(x)$, induced respectively by the entire $n$-layer and the lower tube of the $n$-layer, have the form

$$
E_{n}(x)=\frac{\chi_{n}}{2 \pi k \varepsilon_{m} \cdot x} ; \Delta E_{n l}(x)=\frac{\chi_{n l}(x)}{2 \pi k \varepsilon_{m} \cdot x},
$$

where the linear charge densities $\chi_{n}$ and $\chi_{n l}(x)$ are equal

$$
\chi_{n}=\int_{\rho_{n}}^{\rho_{n+1}} q(\rho) \rho \mathrm{d} \rho \int_{0}^{1} \mathrm{~d} z \int_{0}^{2 \pi} \mathrm{d} \theta ; \quad \chi_{n l}(x)=\int_{\rho_{n}}^{x-\rho_{n}} q(\rho) \rho \mathrm{d} \rho \int_{0}^{1} \mathrm{~d} z \int_{0}^{2 \pi} \mathrm{d} \theta ;
$$

$k=1$ in $\mathrm{CGSE}_{\mathrm{q}} ; k=4 \pi \varepsilon_{0}$ in MKSA. It should be noted here that the fields $E_{n}(x)$ and $\Delta E_{n l}(x)$ are directed from the dislocation line at $q(\rho)-n_{0}>0$, and toward the dislocation line at $q(\rho)-n_{0}<0$ with respect to the photoelectron motion.

An analysis of expressions (10)-(15) shows that, in contrast to the tabulated values of $n_{0}$ [22] for copper, it is necessary to take into account 4 atoms per cell fcc. lattices and $2-3$ valence electrons per atom, in this case the Coulomb forces from point sources of cations and $q(\rho)$ are of the same order $\left[10^{-8} \mathrm{~N}\right]$, which allows us to estimate the height of the potential barrier from the first peak $q(\rho)(n=2)$, which does not exceed $1.2 \mathrm{eV}$, and the depth of the first potential valley $q(\rho)(n=3)$ of the order of $(0.2 \div 0.3) \mathrm{eV}$. The barrier is due to the action of the fields $E_{1}(x)$ and $\Delta E_{2 l}(x)$ from the inside of the peak and the electron interaction $F_{e e}$, whose effective radius is $0.1 \mathrm{~nm}$ [25], which leads the photoelectrons to the first potential valley, where $F_{e e}$ is small and the forces from the fields $E_{2}(x)$ and $E_{3 l}(x)$ are directed inside the valley and create oscillations, and in the presence of the tangential component $v_{\text {phe }}$, they rotate around the dislocation line.

A detailed consideration of the numerical calculation using the Mathcad application, the equations and trajectories of the motion of photoelectrons and donor cations in the nuclei of linear and planar defects is presented in a separate work. We also note that the photoelectron current density $J_{\text {phe }}$ using the dimensional method can be represented as

$$
J_{p h e}=2 \cdot n_{p h e} \cdot e \cdot v_{p h e}=\rho_{d} \cdot \frac{2}{b_{0}} e \cdot v_{p h e},
$$

where $n_{\text {phe }}$ is the density of donor cations in the nuclei of defects; $\rho_{d}$ is the dislocation density $\left[\mathrm{m}^{-2}\right] ; \quad b_{0}=0.71 a_{0} ; v_{\text {phe }}$ is the speed of the photoelectron.

\section{Discussion of the Results}

This transition model allows you to identify a number of fundamentally important features:

1) The existence of a correlation between the components of the tensor of the 
Maxwell stresses of the alternating field $\sigma_{\alpha \beta}^{\text {if }}$ in the core of the defect and the internal elastic stresses $\sigma_{i k}^{e l}$ in its environment.

2) The conversion of the spectral densities $\Psi_{\perp}^{a t}(\beta), \Psi_{\perp}^{e}(\beta), \Psi_{\perp}^{\text {cat }}(\beta)$ as $T \rightarrow 0$ into linear spectra leads to the appearance of threshold values of oscillation currents and rotations of photoelectrons and cations, of which they were knocked out at all stages of plastic deformation and fracture. In this case, intermittent deformation takes place near absolute zero [28], and the asymptote of the exponential envelope of the peaks $\Psi_{\perp}^{a t}(\beta)$ is a straight parallel to the scale $\beta$, which is equivalent to the simultaneous occurrence of translational, rotational plasticity, critical fragmented structures, microcracks, or cold brittleness.

3) The expression of the force acting on the dislocation in the form of $\sigma_{i k} b_{k}$ in the framework of the continuum models is not applicable in the framework of the continuum models in this transition model, because it reflects the change in the elastic energy of the crystal $\Delta \varepsilon_{e l}$ when the dislocation of unit length is displaced by one $a_{0}$, and $\Delta \varepsilon_{e l}$ is several orders of magnitude smaller than the changes energies of the alternating field in the core of a dislocation at similar displacements.

4) At first glance, the Coulomb's static law is not applicable to the description of the defect nucleus, but at an arbitrary time $t \pm \Delta t \geq \hbar / 2 \Delta \varepsilon_{\text {phe }}$ with $v_{\text {phe }} \ll v_{F}$, the distribution $q(\rho)$ creates an additional potential relief $u_{q}(\boldsymbol{r}, t)$, which is adjacent to the main $u(r, t)$ from the Coulomb attraction of the initially immobile cationic chain and essentially "stitches" with it. If the photoelectron moves with velocity $v_{\text {phe }}(t)$ along the $x$ axis, then the expression for the Coulomb force contains the Lorentz correction $\left(1-v_{\text {phe }}^{2}\right)^{-1 / 2}$ times $\left[\frac{\left(x-v_{\text {phe }} \cdot t\right)^{2}}{1-v_{\text {phe }}^{2}}+y^{2}+z^{2}\right]^{-1} \quad$ [[28], p. 259]. At photoelectron velocities $v_{\text {phe }} \leq v_{e}^{\text {thr }}$, a purely quantum phenomenon takes place: a tunnel transition through the first peak $q(\rho)$, where quasistable states in the form of oscillations and rotations arise in the first potential valley $q(\rho)$, which goes from zero to $2 \pi$ in the range of angles $\theta$ from zero to $2 \pi$. Hence, translational plasticity within the framework of discrete and transitional models takes on a new meaning.

5) Generation of nuclei of linear defects involves the formation of linear structures under the influence of an alternating field in the generalized space of rectangular pulses and due to the secondary long-wavelength photons of the field $E_{j}^{i f}$ and the correlation $\sigma_{j k}^{i f}$ and $\sigma_{m n}^{e l}$, elastic deformation and stress fields arise in the surrounding defect core medium, and at a distance from the defect line $\rho_{1}=(0.071 \div 0.087) \mathrm{nm}$ equal to the cation radius [5], the photoelectrons receive kinetic energy and tunnel through the potential barrier of the first peak $q(\rho)$, where their quasistable states in the form of oscillations and rotations, and then the movement of donor cations with subsonic speeds from the defect line to potential interstitial wells from the attraction of oscillating and rotating photoelectrons of the electronic chain. The subsequent penetration of 
photoelectrons into the third potential valley at $\rho_{7}=0.73 \mathrm{~nm}$ is the beginning of the linear defect slip, where at $b_{0}<\rho \leq 2 b_{0}$ donor cations begin to pass from sites to internodes, and at $v_{\text {phe }}=(3 \div 5) \times 10^{5} \mathrm{~m} / \mathrm{s}<v_{F}=1.57 \times 10^{6} \mathrm{~m} / \mathrm{s} \quad$ [[22], p. 260] conduction electrons make constant relaxation tuning, which allows us to approximately assume $q(\rho)$ given in time, and if the dislocation is stationary, then in space. On the one hand, following a chronological ordering, when a defect nucleus is generated, a set of linear structures appears, then elastic fields around the defect nucleus. On the other hand, both the generation and slip of a screw dislocation are qualitatively indistinguishable dynamic processes for which the threshold speed of photoelectrons during sliding $v_{s l}^{\text {thr }}>v_{g}^{\text {thr }}$ is the threshold velocity of photoelectrons during generation.

\section{Conflicts of Interest}

The author declares no conflicts of interest regarding the publication of this paper.

\section{References}

[1] Cottrell, A.H. (1953) Dislocations and Plastic Flow in Crystals. 2th Edition, Clarendon Press, Oxford.

[2] Kelly, A. and Groves, G.W. (1970) Crystallography and Crystal Defects. Longman, London.

[3] Kosevich, A.M. (1981) Physical Mechanics of Real Crystals. Naukova Dumka, Kiev.

[4] Mirkin, L.I. (1968) Physical Foundations of Strength and Plasticity. Publishing House of Moscow State University, Moscow.

[5] Vainshtein, B.K., Fridkin, V.M. and Indenbom, V.L. (1979) Modern Crystallography Vol. 2. Structure of Crystals. Springer, Berlin.

[6] Orlov, A.N., Perevezentsev, V.N. and Rybin, V.V. (1980) Borders of Grains in Metals. Metallurgy, Moscow.

[7] Ke, T.S. (1954) In the Book. The Elasticity and Inelasticity of Metals. Trans. from English. Foreign Litrature, Moscow, 198-222, 223-233.

[8] Smoluchowski, R. (1952) Theory of Grain Boundary Diffusion. Physics Review Journals Archive, 87, 482-487. https://doi.org/10.1103/PhysRev.87.482

[9] Li, J.C.M. (1961) High-Angle Tilt Boundary-A Dislocation Core Model. Journal Applied Physics, 32, 525-541. https://doi.org/10.1063/1.1736036

[10] Mott, N.F. (1948) Slip at Grain Boundaries and Grain Growth in Metals. Proceedings of the Physical Society, 60, 391-394. https://doi.org/10.1088/0959-5309/60/4/309

[11] Croxton, C.A. (1974) Liquid State Physics-A Statistical Mechanical Introduction. Cambridge University Press, Cambridge. https://doi.org/10.1017/CBO9780511753480

[12] Shermergor, T.D. (1977) Theory of Elasticity of Micro-Inhomogeneous Media. Nauka, Moscow.

[13] Busov, V.L. (2019) Dynamic Equations of Evolution in the Nuclei of Linear Defects of Crystalline Materials during the Collision of Solids. Physical Mesomechanics, 22, 91-96. 
[14] Akhiezer, A.I. and Berestetskiy, V.B (1969) Quantum Electrodynamics. Nauka, Moscow.

[15] Busov, V.L. (2009) Dynamic Equations of Evolution in the Field of Fragmentation. Physical Mesomechanics, 12, 17-22.

[16] Busov, V.L. and Mikheenko, D.Y. (2015) On the Mechanism of Destruction of the Rolling Roll. Theoretical Model. Physical Mesomechanics, 18, 72-78.

[17] Honeykombe, R.W.K. (1968) The Plastic Deformation of Metals. Edward Arnold Ltd., Cambridge.

[18] Landau, L.D. and Lifshits, E.M. (1973) Field Theory. Nauka, Moscow.

[19] Panin, V.E., Likhachev, V.A. and Grinyaev, Y.V. (1985) Structural Levels of Deformation of Solids. Nauka Siberian Branch, Novosibirsk.

[20] Rybin, V.V. (1986) Large Plastic Deformation and Fracture of Metals. Metallurgy, Moscow.

[21] Oqurtani, T.O. and Huggins, R.A. (1967) Theory of Electric Field Gradient Due to Conduction Electron Charge Density Redistribution around Screw Dislocation in Metals. Physica Status Solidi (b), 24, 301-311. https://doi.org/10.1002/pssb.19670240130

[22] Kittel, C. (1978) Introduction to Solid State Physics. 4th Edition, John Wiley \& Sons, New York.

[23] Novozhilov, Y.V. and Yappa, Y.P. (1978) Electrodynamics. Nauka, Moscow.

[24] Ziman, J.M. (1972) Principles of the Theory of Solids. 2th Edition, Cambridge University Press, Cambridge. https://doi.org/10.1017/CBO9781139644075

[25] Reims, S. (1972) Theory of Many-Electron Systems. North-Holland Publishing Company, Amsterdam.

[26] Kikoin, I.K. (1976) Tables of Physical Quantities. Atomizdat, Moscow.

[27] Kuzmichev, V.E. (1989) Laws and Formulas of Physics. Naukova Dumka, Kiev.

[28] Feynman, R., Leighton, R. and Sands, M. (1964) Feynman Lectures on Physics Electrodynamics Vol. 2. Addison Wesley Publishing Company, Inc., Massachusetts. 